\title{
Effects of high-fat diet on 1,2-dimethylhydrazine-induced aberrant crypt foci and colorectal tumours in rats
}

\author{
GUANGYING QI ${ }^{1,2^{*}}$, BO TANG $^{3^{*}}$, LIHUA ZHOU $^{1,2^{*}}$, HIROSHI JIKIHARA $^{2}$, ATSUMI KIWATA $^{2}$, \\ YUKI SAKAMOTO ${ }^{2}$, FANG TANG ${ }^{1}$, SHENGJUN XIAO $^{1}$, ZHENRAN WANG $^{3}$, QIUHUI WU $^{1}$, \\ HUILING LU ${ }^{1}$, ZHEN WU ${ }^{1}$, SIEN ZENG ${ }^{1}$ and FUMIO SHIMAMOTO ${ }^{2}$ \\ ${ }^{1}$ Department of Pathology and Physiopathology, Guilin Medical University, Guilin, Guangxi 541004, P.R. China; \\ ${ }^{2}$ Department of Health Sciences, Faculty of Human Culture and Science, Prefectural University of Hiroshima, \\ Hiroshima 734-8558, Japan; ${ }^{3}$ Department of Hepatobiliary and Gastrointestinal Surgery, \\ Affiliated Hospital of Guilin Medical University, Guilin, Guangxi 541001, P.R. China
}

Received January 5, 2015; Accepted February 2, 2015

DOI: $10.3892 /$ br.2015.433

\begin{abstract}
Obesity is one of the leading causes of numerous types of cancer. The present study investigated the impact of a high-fat diet on 1,2-dimethylhydrazine (DMH)-induced colorectal cancer (CRC) in F344 rats. A total of 16 male F344 rats aged 4 weeks were randomly divided into two groups (8 rats/group). Rats in group A were fed a basal diet with a moderate fat (MF) content, while rats in group B were fed a high-fat diet. Upon reaching 5 weeks of age, the rats were injected subcutaneously with DMH (20 mg/kg body weight). $\mathrm{DMH}$ was administered once a week for 8 consecutive weeks. All the rats were sacrificed 34 weeks after the first $\mathrm{DMH}$ injection and dissected to obtain samples of colorectal tissues. The tissues were examined under a microscope for the presence of aberrant crypt foci (ACFs) and subjected to histopathological analysis. The results showed that at the end of the 34-week experiment, body weights and visceral fat levels were significantly higher in the high-fat diet group compared to the basal diet group. In addition, the incidences of colorectal $\mathrm{ACF}$, adenoma and adenocarcinoma were markedly elevated in the high-fat diet group compared to the basal diet group. These results indicate that the consumption of a high-fat diet promotes the development and progression of CRC and the control of fat intake may prevent CRC.
\end{abstract}

Correspondence to: Professor Sien Zeng, Department of Pathology and Physiopathology, Guilin Medical University, 109 North 2nd Huan Cheng Road, Guilin, Guangxi 541004, P.R. China

E-mail: zse@glmc.edu.cn

Professor Fumio Shimamoto, Department of Health Sciences, Faculty of Human Culture and Science, Prefectural University of Hiroshima, 1-1-71 Ujina-Higashi, Hiroshima 734-8558, Japan

E-mail: simamoto@pu-hiroshima.ac.jp

*Contributed equally

Key words: high-fat diet, antitumour, colorectal cancer, F344 rats

\section{Introduction}

Colorectal cancer (CRC) is the second and third most common malignancy in females and males, respectively, worldwide. Colon cancer is considered to be one of the leading causes of mortality in the world. There are extremely few therapeutic agents capable of effectively treating colon cancer (1). According to the GLOBOCAN database established by the World Health Organization (WHO), an estimated 12.7 million new cancer cases and 7.6 million cancer fatalities occurred in 2008 (2). The incidence of CRC is rising rapidly in numerous Asian countries and approaching the incidence in developed countries (3). Studies have shown that the incidence of malignant tumours has declined in males and females in the United States and one of the notable contributing factors is the decreased incidence of $\mathrm{CRC}$. The results indicate that $\mathrm{CRC}$ occupies a predominant position among the malignancies in the United States (4). African-Americans have experienced an increased incidence of CRC compared to other U.S. populations. In addition, the prevalence of proximal CRC is higher among African-Americans and the average age for the onset of CRC is becoming younger (5). CRC is one of the common malignancies in China. The mortality rate of CRC in China is 4.54 per 100,000 population, which accounts for $4.9 \%$ of all cancer fatalities and ranks as the fifth highest among the malignant tumours. Notably, thus far the incidence and mortality rate of $\mathrm{CRC}$ continues to exhibit a tendency to increase. The five-year survival rates of CRC after curative resection remain near $50 \%$ (for rectal cancer) and $70 \%$ (for colon cancer). CRC is closely associated with the lifestyle of Western countries. Lifestyle and dietary patterns affect the risk of colon cancer. Among dietary factors, high intakes of red meat, fat and carbohydrates enhance the risk of developing CRC. By contrast, increased consumption of fruits, vegetables and fibres may reduce the incidence of CRC (6-8). The report released by the WHO and the Food and Agriculture Organization of the United Nations (FAO) in 2003 clearly stated that obesity is a risk factor for CRC (9). 1,2-Dimethylhydrazine (DMH) is a carcinogenic agent widely utilised to induce CRC in rats and other animal models. DMH is also commonly employed in 
chemistry-based cancer prevention studies (10). The present study mainly investigated the effects of a high-fat diet on DMH-induced CRC in F344 rats.

\section{Materials and methods}

Animals. A total of 16 male 4-week-old F344 rats were purchased from Charles River Laboratories Japan, Inc. (Kanagawa, Japan). The animals were cared for in compliance with the principles and guidelines of Ethical Committee for Animal Care and Institutional Animal Ethical Committee, in accordance with the Japan National Law on Animal Care and Use. The Ethical Committee for Animal Care of the Prefectural University of Hiroshima (Hiroshima, Japan) approved the experiments undertaken. The rats were housed in a standard air-conditioned room at the Laboratory Animal Research Center of the Prefectural University of Hiroshima. The room provided a controlled ambient temperature of $23 \pm 2^{\circ} \mathrm{C}$, a humidity of $50 \pm 10 \%$ and a daily light period of $12 \mathrm{~h}$. The rats had free access to drinking water and were fed either a basal diet [moderate fat (MF)] or a high-fat diet (Oriental Yeast Co., Ltd., Tokyo, Japan). The main composition of the high-fat diet included lard, a fish meal, defatted soybean, vitamins and minerals (Table I). The contents of the three major types of nutrients in the high-fat and basal diets were compared and summarised in Table II. The contents of proteins, fats and carbohydrates were $60,24.5$ and $7.5 \mathrm{~g}$, respectively, in $100 \mathrm{~g}$ of the high-fat diet and 23.6, 5.3 and $54.4 \mathrm{~g}$, respectively, in the basal diet. The body weights of the rats were recorded once every two weeks. DMH was purchased from Tokyo Chemical Industry Co., Ltd., (Tokyo, Japan).

Experimental protocol. The experimental design is shown in Fig. 1. The 16 male rats were randomly divided into two groups (8 rats/group) based on their diets: Group A, basal diet; group B, high-fat diet. $\mathrm{DMH}$ was dissolved in $0.9 \%$ sodium chloride and the $\mathrm{pH}$ value was adjusted to 6.5 with $\mathrm{NaHCO}_{3}$. Upon reaching 5 weeks of age, the experimental rats were injected subcutaneously with DMH ( $20 \mathrm{mg} / \mathrm{kg}$ body weight $)$ once a week for 8 consecutive weeks. All the rats were sacrificed 34 weeks after the first DMH injection and dissected to obtain samples of colorectal tissues. The tissues were examined under a microscope for the presence of aberrant crypt foci (ACFs) and subjected to histopathological analysis.

Analysis of ACFs and histological analysis. In rodents and humans, ACFs are believed to be the microscopic damage
Table I. Contents of the high-fat diet.

\begin{tabular}{lr} 
Nutrient composition of the high-fat diet & $\%$ \\
\hline Lard & 58 \\
Fish meal & 30 \\
Defatted soybean & 10 \\
Vitamins, minerals and other components & 2
\end{tabular}

Table II. Contents of the three major types of nutrients in the basal and high-fat diets (100 g).

\begin{tabular}{lcc}
\hline Nutrients & Basal diet & High-fat diet \\
\hline Protein, g & 23.6 & 24.5 \\
Fat, $g$ & 5.3 & 60 \\
Carbohydrate, g & 54.4 & 7.5 \\
Total energy, kcal & 360 & 640 \\
\hline
\end{tabular}

formed at the earliest stage of CRC development. ACFs may be the precancerous lesions that emerge prior to dysplasia (11). DMH-induced intestinal mucosal injury is a multi-step pathological process that involves the formation of ACFs, a gradual increase in the number of ACFs and eventually the development of CRC (12). The emergence of ACFs is closely associated with DMH. DMH is converted into azoxymethanol in vivo, which is further decomposed to form alkylated methyl diazonium. The product induces the methylation of DNA, RNA and proteins. DNA methylation results in chromosomal gene mutations and tumour cell formation. A study conducted by Tanaka (13) has shown that the DNA sequence of proto-oncogene $\mathrm{K}$ is mutated in ACFs, leading to the formation of adenomas. Subsequently, DNA repair errors and p53 gene mutation occur and adenomas develop further into adenocarcinomas.

After the animals were sacrificed, the entire colorectum was quickly removed and cut open along the longitudinal axis. The colorectal samples were rinsed clean with precooled $0.9 \%$ $\mathrm{NaCl}$ solution and laid onto filter paper with the mucosal side up. After $24 \mathrm{~h}$ of fixation at $4^{\circ} \mathrm{C}$ in $10 \%$ buffered formalin, the colorectal samples were stained with $0.5 \%$ methylene blue for 15-30 min, washed with distilled water, placed on glass slides and observed under an optical microscope using the 4 and $10 \mathrm{X}$ objectives. The number of ACFs was recorded. After the

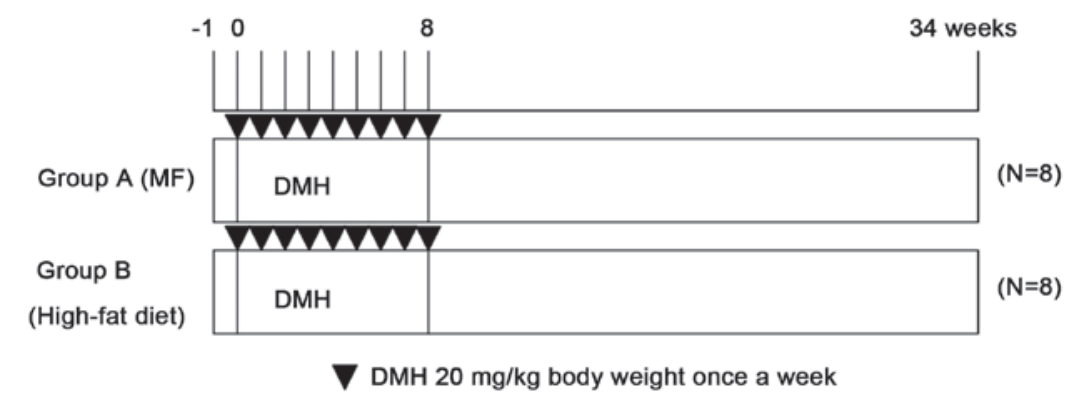

Figure 1. Experiment protocol. MF, moderate fat; DMH, 1,2-dimethylhydrazine. 
Table III. Number of colon adenomas.

No. of colon adenomas

Group Mild-grade dysplasia Moderate-grade dysplasia Severe-grade dysplasia Total No. of colon adenomas per rat

\begin{tabular}{llllll}
\hline A & 103 & 16 & 0 & 118 & $14.8 \pm 8.54^{\mathrm{a}}$ \\
$\mathrm{B}$ & 141 & 30 & 4 & 175 & $21.9 \pm 7.19$ \\
\hline
\end{tabular}

${ }^{\text {ap }}<0.01$

Table IV. Number of colon adenocarcinomas per rat.

\begin{tabular}{|c|c|c|c|c|c|}
\hline \multirow[b]{2}{*}{ Group } & \multicolumn{4}{|c|}{ No. of colon adenocarcinomas } & \multirow[b]{2}{*}{$\begin{array}{c}\text { No. of colon } \\
\text { adenocarcinomas per rat }\end{array}$} \\
\hline & $\begin{array}{l}\text { Well-differentiated } \\
\text { adenocarcinomas }\end{array}$ & $\begin{array}{l}\text { Moderately differentiated } \\
\text { adenocarcinomas }\end{array}$ & $\begin{array}{l}\text { Poorly differentiated } \\
\text { adenocarcinomas }\end{array}$ & Total & \\
\hline A & 4 & 1 & 3 & 8 & $1.0 \pm 0.83^{\mathrm{a}}$ \\
\hline B & 16 & 3 & 1 & 20 & $2.5 \pm 1.07$ \\
\hline
\end{tabular}

${ }^{\mathrm{a}} \mathrm{P}<0.01$.

ACFs were recorded and calculated, the colorectal samples were fixed with $10 \%$ buffered formalin, embedded in paraffin, sectioned at a thickness of $4 \mu \mathrm{m}$, stained with hematoxylin and eosin and examined under an optical microscope (Olympus, Tokyo, Japan). The number of tumours was counted and tumour types and grades were investigated.

Statistical analysis. The Statcel software package (KaleidaGraph version 4.1) was used for analysis. The Fisher's exact test and t-test (Statcel, the useful add in forms on Excel; 2nd edition) were used to compare data between the two groups. $\mathrm{P}<0.05$ was considered to indicate a statistically significant difference.

\section{Results}

Body weight and visceral fat content. Fig. 2A shows no significant differences in body weight between the two groups of rats within the first 14 weeks of the experiment. However, compared to the rats in the basal diet group, the rats in the high-fat diet group experienced significantly greater weight gains after 14 weeks. At the end of the 34-week experiment, body weights of the rats in the high-fat diet group were significantly higher compared to the rats in the basal diet group $(418.6 \pm 14.9$ vs. $381.9 \pm 20.9 \mathrm{~g} ; \mathrm{P}<0.05)$. For the purpose of the study, the amount of visceral fat was calculated as the sum of the amount of body fat deposited in the greater omentum and testicles. The amounts of visceral fat in the two groups of rats were determined and are shown in Fig. 2B. The amount of visceral fat was significantly increased in the high-fat diet group compared to that of the basal diet group (19.2 \pm 1.9 vs. $13.1 \pm 1.9 \mathrm{~g} ; \mathrm{P}<0.01)$. The average daily energy intake in each rat from the high-fat diet group was $\sim 1$.3-fold higher compared to that of the basal diet group and the average daily fat intake in each rat from the high-fat diet group increased $\sim 8$-fold compared to that of the basal diet group (data not shown).

Colonic ACFs. The effect of the tested diets on the growth and development of DMH-induced ACFs in rats is shown in Fig. 3. All the rats treated with DMH showed a $100 \%$ incidence. The high-fat diet group showed a significantly higher average number of ACFs than that of the MF diet group.

Colon tumours. The colorectal tissue sections were subjected to histopathological investigation and the results are summarised in Tables III and IV, and Fig. 4. Tables III and IV show that at the 34th week of the experiment, the incidence of colorectal adenomas and adenocarcinomas was significantly higher in the high-fat diet group compared to that of the basal diet group. A total of 119 adenomas were detected in the basal diet group, including 103 low-grade and 16 moderate-grade adenomas. The average number of adenomas developed in each rat was $14.8 \pm 8.5$. By contrast, 175 adenomas were detected in the high-fat diet group, including 141 low-grade, 30 moderate-grade and 4 high-grade adenomas. The average

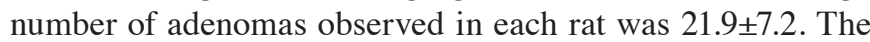
differences in the adenoma incidence between the two groups of rats were statistically significant $(\mathrm{P}<0.01)$. In addition, the numbers of highly, moderately and poorly differentiated adenocarcinomas found in the basal diet group were 4, 1 and 3, respectively. An average of $1.0 \pm 0.8$ adenocarcinomas were developed in each rat. By contrast, the numbers of highly, moderately and poorly differentiated adenocarcinomas detected in the high-fat diet group were 16, 3 and 1, respectively. An average of $2.5 \pm 1.1$ adenocarcinomas were developed in each rat. The differences in the adenocarcinoma incidence between the two groups of rats were also statistically significant $(\mathrm{P}<0.01)$. 

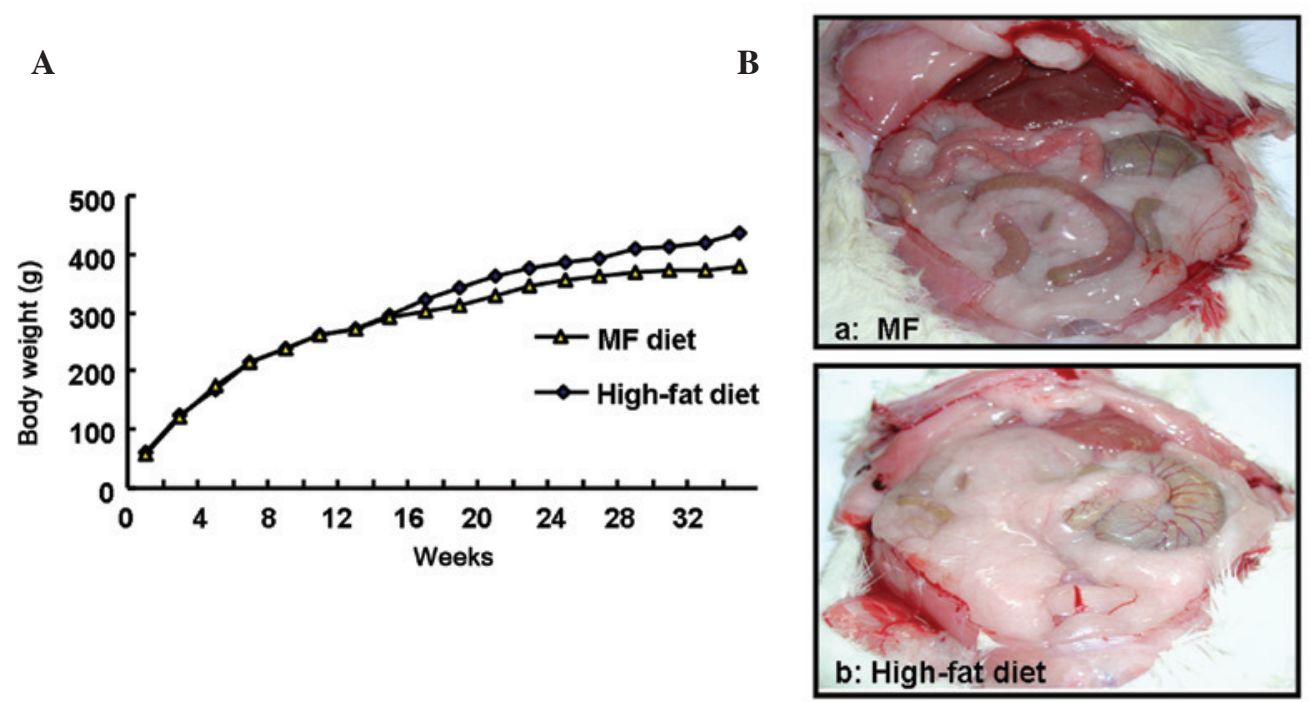

C

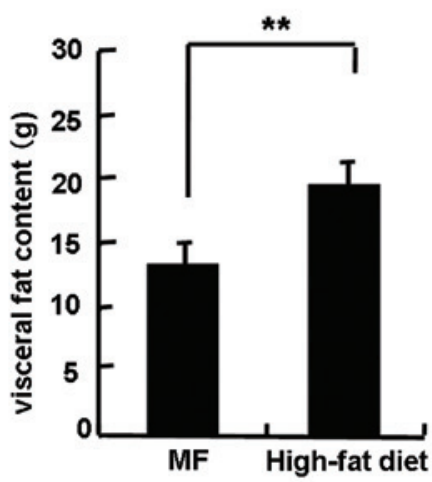

Figure 2. Body weights and visceral fat content of the different groups of F344 rats throughout the experiment. (A) The body weights of the different groups of F344 rats. (B) Visceral image of the different groups of rats; (a) moderate fat (MF) and (b) high-fat diet groups. (C) Visceral fat content of the different groups of F344 rats, ${ }^{* *} \mathrm{P}<0.01$.

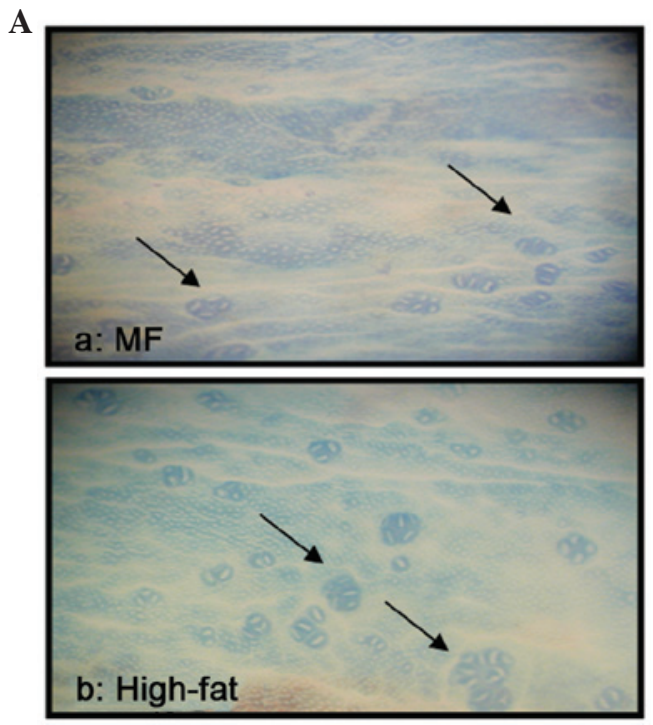

B

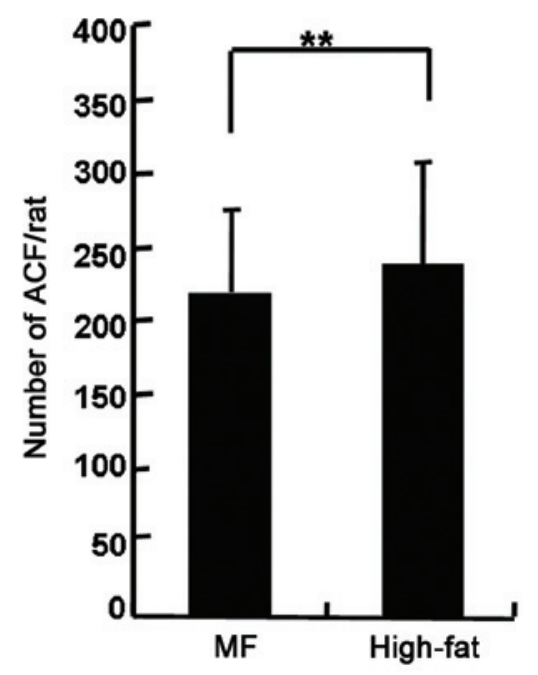

Figure 3. Aberrant crypt foci (ACFs) expression in the colon of rats treated with 1,2-dimethylhydrazine (DMH) and stained with $0.5 \%$ methylene blue at week 34. (A) ACF expression in the colon of rats treated with DMH and stained with $0.5 \%$ methylene blue. (a) ACF expression in the colon from a rat in the modified fat (MF) group. Arrows point to the four and five crypts in the colon. (b) ACF expression in the colon of a rat in the high-fat diet group. Arrows point to the multiple crypts in the colon. (B) Number of ACFs in the colon, ${ }^{* * *} \mathrm{P}<0.01$.

\section{Discussion}

Obesity is one of the leading causes of numerous types of cancer. The report released by the WHO and the FAO in 2003 clearly stated that obesity is a risk factor for CRC (9). Investigators in the U.S. have conducted studies on the potential link between obesity and cancer and found that in males and females, kidney cancer and cancers of the digestive system, such as oesophageal cancer, CRC, hepatobiliary and pancreatic cancers, are associated with obesity, as defined by high body mass index values (14). Relevant studies have shown that obese populations with high blood sugar levels have almost double the risk of developing obesity-related cancers, while obese populations with normal blood sugar levels only carry a
$50 \%$ increased risk of developing cancers. In addition, metabolic dysfunction also increases the risk of cancer in obese individuals (15). In the present study, CRC was induced in rats using DMH. The rats were fed a high-fat diet and the effect of the high-fat diet-induced obesity on rat CRC was investigated. The results showed that after 14 weeks, the rats in the high-fat diet group experienced a significantly greater body weight gain compared to the rats in the basal diet group. At the end of the 34-week experiment, the average difference in body weights between the two groups of rats was $\sim 40 \mathrm{~g}$. The level of visceral fat was significantly higher in rats from the high-fat diet group compared to the rats from the basal diet group. In addition, the incidence of ACF, adenomas and adenocarcinomas was markedly elevated in the high-fat diet group compared to the 
A

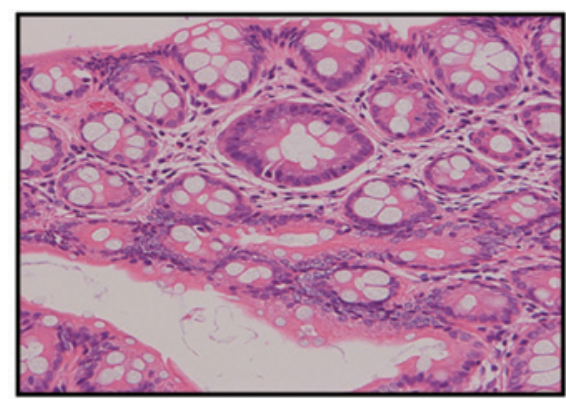

C

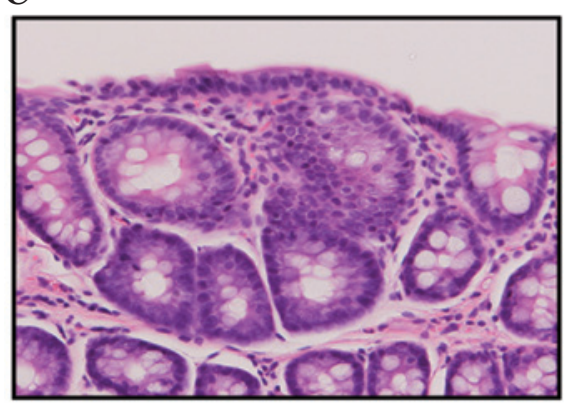

E

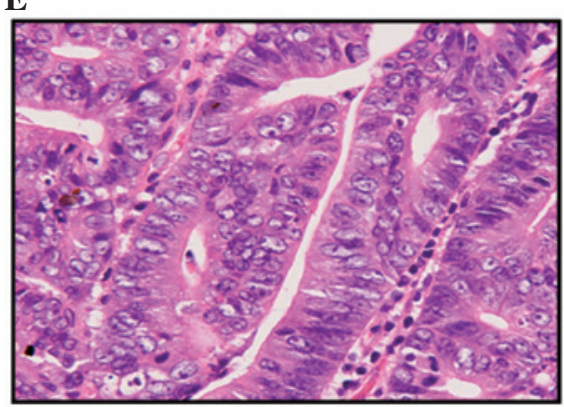

B

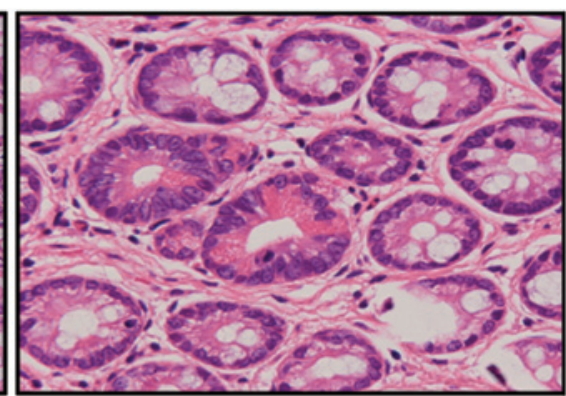

D

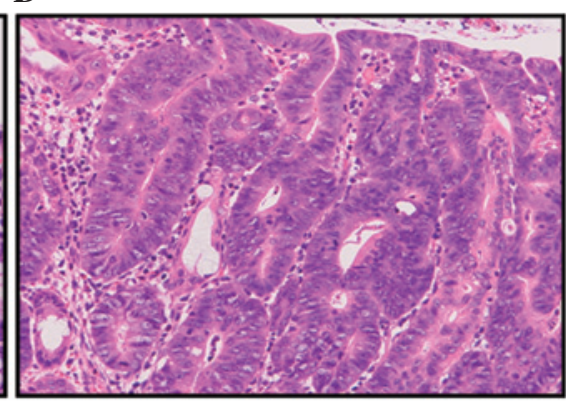

F

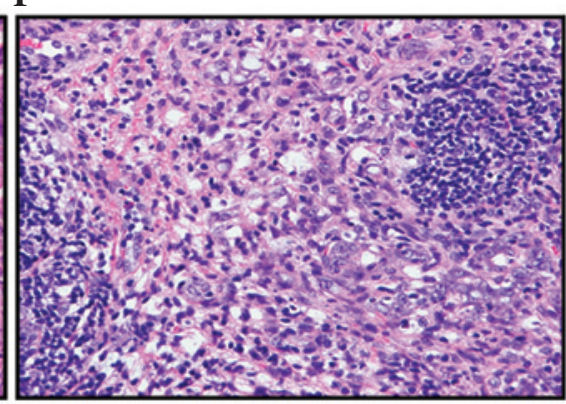

Figure 4. 1,2-Dimethylhydrazine-induced colorectal tumours in rats. (A) Hyperplasia. (B) Adenoma with mild atypia. (C) Adenoma with moderate atypia. (D) Well-differentiated tubular adenocarcinomas. (E) Moderately differentiated adenocarcinomas. (F) Poorly differentiated adenocarcinomas. Haematoxylin and eosin stains (magnification, x200).

basal diet group. The above results indicate that a high-fat diet promotes the development and progression of CRC in rats.

The mechanisms by which obesity induces CRC may be associated with insulin-resistant hyperinsulinaemia and insulin-like growth factors (IGFs) $(14,16)$. To maintain normal blood sugar levels and carbohydrate metabolism, patients with insulin resistance require a large amount of insulin. A variety of conditions, such as obesity, inflammation and hyperlipidaemia, may result in the loss of normal insulin function. Obesity causes insulin resistance and hyperinsulinaemia. Shortly following the inhibition of insulin, the secretion and expression of IGF binding protein are altered, which increases the amount of free IGFs. Insulin and IGF-1 are growth factors that promote cell proliferation and inhibit apoptosis in cancer cells, resulting in tumour development and progression. Blood insulin levels measured in CRC patients and healthy individuals before breakfast and at 90 minutes after breakfast or lunch have shown that an increased IGF-1 level is associated with the incidence of CRC (17). In addition, the levels of C-peptide have been analysed in CRC patients and healthy individuals. The group with the highest $\mathrm{C}$-peptide value exhibits a 3.2-fold increase in CRC risk compared to the group with the lowest $\mathrm{C}$-peptide value. Higher C-peptide values indicate a greater risk of CRC $(17,18)$.
In addition to insulin resistance, high-fat diet-induced bile acid secretion also promotes CRC development. Increased animal fat intake enhances the levels of secreted bile acids and cholesterol in bile. Bile acids and cholesterol are converted to secondary bile acids and steroids by bacteria in the colon, which are further metabolised by colonic bacteria into carcinogens (19). Animal experiments have shown that a high-fat diet increases bile acid secretion. Bile acids exhibit no direct carcinogenic effects; however, they increase the rate of cancer induction by carcinogens through accelerating cell turnover (20). This study showed that the consumption of a high-fat diet significantly increased the incidence of colorectal adenomas and adenocarcinomas, indicating that the cancer-promoting effect of a high-fat diet is associated with the increased secretion of bile acids. In addition, bile acids induce the expression of cyclooxygenase-2 (Cox-2) in CRC cells (21). Cox-2 is rarely expressed in the majority of normal tissues but is highly expressed in cancer cells. Cox-2 inhibits apoptosis in cancer cells and promotes angiogenesis (21-23). In addition, secondary bile (docosahexaenoic acid) triggers the production of the receptor of urokinase-type activating factor and is associated with the increased invasiveness of CRC cells (24). 
Obesity is a strong risk factor for CRC. Adiponectin (APN) is a lipid factor secreted by adipose tissue that exhibits an immune-modulatory, anticancer effect in carbohydrate and lipid metabolism in CRC. A number of studies have shown that obesity is associated with APN and the incidence of CRC (25-29). Wei et al (30) found that the level of APN was reduced in obese individuals. APN deficiency induces chronic inflammation of the large intestine, which may further develop into CRC and increase the risk of developing cancer. Saxena et al (31) have investigated DMH-induced CRC under the condition of APN deficiency and identified that experimental animals deficient in APN clearly exhibit the clinical symptoms of carcinogenesis.

The consumption of a high-fat diet induces insulin resistance and increases bile secretion. The products of lipid metabolism exhibit mutagenic effects on DNA and RNA. In addition, the APN level is reduced in individuals with a high body fat level. Therefore, a high-fat diet promotes the development of CRC. Exercise may have a preventive effect against CRC. It is also necessary to maintain a nutritionally balanced diet and avoid excess fat intake. The present study also showed that the consumption of a high-fat diet promoted the development and progression of CRC. Therefore, controlling fat intake may prevent CRC.

\section{Acknowledgements}

The present study was supported in part by the Important Research Grant from the Prefectural University of Hiroshima, Hiroshima Tsuchiya General Hospital. The authors thank them for their technical and materials assistance.

\section{References}

1. Misra S, Ghatak S, Vyas A, et al: Isothiocyanate analogs targeting CD44 receptor as an effective strategy against colon cancer. Med Chem Res 23: 3836-3851, 2014.

2. Ferlay J, Shin HR, Bray F, Forman D, Mathers C and Parkin DM: Estimates of worldwide burden of cancer in 2008 GLOBOCAN 2008. Int J Cancer 127: 2893-2917, 2010.

3. Chung SJ, Kim YS, Yang SY, Song JH, Park MJ, Kim JS, JungHC and Song IS: Prevalence and risk of colorectal adenoma in asymptomatic Koreans aged 40-49 years undergoing screening colonoscopy. J Gastroenterol Hepatol 25: 519-525, 2010

4. Jemal A, Siegel R, Xu J and Ward E: Cancer statistics, 2010. CA Cancer J Clin 60: 277-300, 2010.

5. Xicola R, Gagnon M, Clark JR, et al: Excess of proximal microsatellite-stable colorectal cancer in African Americans from a multiethnic study. Clin Cancer Res 20: 4962-4970, 2014.

6. Chan AT and Giovannucci EL: Primary prevention of colorectal cancer. Gastroenterology 138: 2029-2043, 2010.

7. Willett WC, Stampfer MJ, Colditz GA, Rosner BA and Speizer FE: Relation of meat, fat, and fiber intake to the risk of colon cancer in a prospective study among women. N Engl J Med 323: 1664-1672, 1990.

8. Macdonald RS and Wagner K: Influence of dietary phytochemicals and microbiota on colon cancer risk. J Agric Food Chem 60: 6728-6735, 2012

9. Amine EK, Baba NH, Belhadj M, et al: Diet, nutrition and the prevention of choronic diseases. World Health Organ Tech Rep Ser 916: 1-149, 2003.

10. Baskar AA, Ignacimuthu S, Paulraj GM and Al Numair KS: Chemopreventive potential of beta-Sitosterol in experimental colon cancer model - an in vitro and in vivo study. BMC Complement Altern Med 10: 24, 2010.
11. Inamine $M$, Suzui $M$, Morioka $T$, Kinjo $T$, Kaneshiro T, Sugishita T, Okada T and Yoshimi N: Inhibitory effect of dietary monoglucosylceramide 1-O-beta-glucosyl-N-2'-hydroxyarachidoyl-4,8-sphingadienine on two different categories of colon preneoplastic lesions induced by 1,2-dimethylhydrazine in F344 rats. Cancer Sci 96: 876-881, 2005.

12. Rodrigues MA, Silva LA, Salvadori DM, De Camargo JL and Montenegro MR: Aberrant crypt foci and colon cancer: Comparison between a short- and medium-term bioassay for colon carcinogenesis using dimethylhydrazine in Wistar rats. Braz J Med Biol Res 35: 351-355, 2002.

13. Tanaka T: Development of an inlammation-associated colorectal cancer model and its application for research on carcinogenesis and chemoprevention. Int J Inflamm 2012: 1-16, 2012.

14. Frezza EE, Wachtel MS and Chiriva-Internati M: Influence of obesity on the risk of developing colon cancer. Gut 55: 285-291, 2006.

15. Moore LL, Chadid S, Singer MR, Kreger BE and Denis GV: Metabolic health reduces risk of obesity-related cancer in framingham study adults. Cancer Epidemiol Biomarkers Prev 23: 2057-2065, 2014.

16. Giovannucci E: Insulin, insulin-like growth factors and colon cancer: A review of the evidence. J Nutr 131 (Suppl 11): 3109S-3120S, 2001.

17. Otani T, Iwasaki M, Sasazuki S, et al: Plasma C-peptide, insulin-like growth factor-I, insulin-like growth factor binding proteins and risk of colorectal cancer in a nested case-control study: The Japan public health center-based prospective study. Int J Cancer 120: 2007-2012, 2007.

18. Powell AA, LaRue JM, Batta AK and Martinez JD: Bile acid hydrophobicity is correlated with induction of apoptosis and/or growth arrest in HCT116 cells. Biochem J 356: 481-486, 2001.

19. Hill MJ and Aries VC: Faecal steroid composition and its relationship to cancer of the large bowel. J Pathol 104: 129-139, 1971.

20. Ryser HJP: Chemical carcinogenesis. N Engl J Med 285: 721-734, 1971.

21. Oshio $\mathrm{H}$, Abe $\mathrm{T}$, Onogawa $\mathrm{T}$, et al: Peroxisome proliferator-activated receptor alpha activates cyclooxygenase- 2 gene transcription through bile acid transport in human colorectal cancer cell lines. J Gastroenterol 43: 538-549, 2008.

22. Wang D, Wang H, Shi Q, Katkuri S, Walhi W, Desvergne B, Das SK, Dey SK and DuBois RN: Prostaglandin E(2) promotes colorectal adenoma growth via transactivation of the nuclear peroxisome proliferator-activated receptor delta. Cancer Cell 6: 285-295, 2004.

23. Tsujii M, Kawano S, Tsuji S, et al: Cyclooxygenase regulates angiogenesis induced by colon cancer cells. Cell 93: 705-716, 1998.

24. Baek MK, Park JS, Park JH, Kim MH, Kim HD, Bae WK, Chung IJ, Shin BA and Jung YD: Lithocholic acid upregulates uPAR and cell invasiveness via MAPK and AP-1 signaling in colon cancer cells. Cancer Lett 290: 123-128, 2010.

25. Larsson SC and Wolk A: Obesity and colon and rectal cancer risk: A meta-analysis of prospective studies. Am J Clin Nutr 86: 556-565, 2007.

26. Gunter MJ and Leitzmann MF: Obesity and colorectal cancer: Epidemiology, mechanisms and candidate genes. J Nutr Biochem 17: 145-156, 2006

27. Calle EE and Kaaks R: Overweight, obesity and cancer: Epidemiological evidence and proposed mechanisms. Nat Rev Cancer 4: 579-591, 2004.

28. Birmingham JM, Busik JV, Hansen-Smith FM and Fenton JI: Novel mechanism for obesity-induced colon cancer progression. Carcinogenesis 30: 690-697, 2009.

29. Bianchini F, Kaaks R and Vainio H: Overweight, obesity, and cancer risk. Lancet Oncol 3: 565-574, 2002.

30. Wei EK, Giovannucci E, Fuchs CS, Willett WC and Mantzoros CS: Low plasma adiponectin levels and risk of colorectal cancer in men: A prospective study. J Natl Cancer Inst 97: 1688-1694, 2005.

31. Saxena A, Chumanevich A, Fletcher E, Larsen B, Lattwein K, Kaur K and Fayad R: Adiponectin deficiency: Role in chronic inflammation induced colon cancer. Biochim Biophys Acta 1822: $527-536,2012$. 